Open Access

\title{
Laparoscopic resection of retroperitoneal lymphangioma around the pancreas: a case report and review of the literature
}

\author{
Takafumi Sato, Yoichi Matsuo*, Kazuyoshi Shiga, Kenta Saito, Mamoru Morimoto, Hirotaka Miyai
} and Hiromitsu Takeyama

\begin{abstract}
Introduction: Lymphangiomas are rare, benign tumors. An intra-abdominal location of these lesions is rarer still and there are only a few reports describing laparoscopic resection for retroperitoneal lymphangiomas, especially in tumors that mimic pancreatic tumors.

Case presentation: We present the case of an asymptomatic 30-year-old Japanese woman in whom a cystic tumor was found incidentally in close approximation to the pancreas. Because the tumor was located in the retroperitoneal space and the body of the pancreas was compressed, we were unable to distinguish a cystic lymphangioma from cystic pancreatic tumors. We started the procedure laparoscopically with five ports. The tumor was in fact separated from the pancreas and was dissected free from the body of the pancreas using scissors and laparoscopic coagulating shears. The left gastric vessels, which were compressed by the tumor, were preserved. As we realized that the tumor was connected to the retroperitoneal lymphatic tissue, we completed the procedure by performing a cystectomy without rupture. The specimen was extracted using a plastic bag. Our patient was discharged on postoperative day 7 without any complications. There is no evidence of recurrence during a $>2$-year observation period.
\end{abstract}

Conclusions: In addition to the therapeutic significance in differentiating between a cystic lymphangioma in close approximation to the body of the pancreas and a pancreatic cystic neoplasm, the laparoscopic approach is feasible and effective.

Keywords: Cystic lymphangioma, Retroperitoneum, Laparoscopy

\section{Introduction}

Lymphangiomas are rare and benign tumors, which are caused by a malformation or a blockage of lymphatic vessels [1]. While in most cases the lesions are located superficially on the body, on rare occasions they can occur in the retroperitoneal space $[2,3]$. There are two types of lymphangiomas, namely cystic and cavernous. If the tumor is located adjacent to the pancreas, it is very difficult to diagnose through preoperative imaging alone [4]. Because recurrence after drainage of a cystic lymphangioma has been reported [5], complete surgical resection is preferred for a cure [1]. However, some authors argue

\footnotetext{
* Correspondence: matsuo@med.nagoya-cu.ac.jp

Department of Gastroenterological Surgery, Nagoya City University Graduate School of Medical Sciences, 1, Kawasumi, Mizuho-cho, Mizuho-ku, Nagoya
} 467-8601, Japan

\section{Case presentation} chronic cough and had a workup in the interna emia. A chest computed tomography scan showed a cys lesion attached to the body of her pancreas, and a diagnosis of cystic pancreatic tumor was made. Blood tests showed neither inflammation nor anemia. Her serum amylase, lipase, aminotransferase, and bilirubin levels were within normal limits. Tumor markers including CEA, 
CA19-9, and AFP were not elevated. A contrast-enhanced computed tomography scan of her abdomen showed a 5 $\mathrm{cm}$ tumor (Fig. 1). The lesion was attached to and compressing the body of the pancreas, the left gastric artery, the body of the stomach, and the abdominal part of the esophagus superiorly. The wall of the mass was thin and not enhanced. Magnetic resonance images showed a multilocular tumor. Neither an internal nodule nor septations were noted. There was no abnormality in the pancreatic duct, and the main pancreatic duct was not in communication with the lesion. Endoscopic ultrasonography showed that the cyst content had iso- and hypoechoic density with a thin wall and no mural nodules. A fine-needle aspiration biopsy was not performed. Differential diagnosis included a mucinous cystic neoplasm, a pancreatic cyst, or a retroperitoneal cystic lymphangioma. She was thus referred to the surgical department for evaluation. At this stage, we could not determine whether a pancreatectomy was necessary, thus we proceeded with a diagnostic laparoscopy.

The surgery was performed 1 month after the first visit to our hospital. Under general anesthesia, the operation was started laparoscopically with five ports $(5 \mathrm{~mm} \times 3$ and $12 \mathrm{~mm} \times 2$ ). The tumor was within the lesser sac, which was incised and a window created. Because the left lateral segment of the liver was large and obscured the surgical view, we added a 5-mm port to elevate the lateral segment. The tumor was located in the retroperitoneal space and was attached to the body of the pancreas. We began the dissection of the tumor from the opposite side of the pancreas using laparoscopic coagulation shears (LCS, Harmonic ACE; Ethicon Endo-Surgery, Cincinnati, $\mathrm{OH}, \mathrm{USA}$ ) and scissors. Branch vessels from the left gastric artery and vein were divided and the compressed left gastric vessels were preserved. The dissection was done easily and bluntly. We dissected the tumor from the pancreas gently using a lymph node dissection technique similar to that in gastric cancer, and were able to separate the mass from the pancreas. Eventually, the tumor was connected only to retroperitoneal lymphatic tissue around the celiac axis. At this stage it became clear that this was a case of cystic lymphangioma; as such, we finished the operation with cystectomy only. The specimen was extracted using a plastic bag (Endo-pouch; Ethicon Endo-Surgery). An additional movie file shows this image in more detail (see Additional file 1). The postoperative course was uneventful and our patient was discharged on the sixth postoperative day. As the postoperative follow-up, our patient received an abdominal computed tomography examination at 3 months after surgery and then every 6 months. Our patient had no signs of recurrence for a 2 -year period after the surgery.

A histopathological examination showed that the cyst was lined by flattened endothelium. Immunostaining was positive for CD31, D2-40, AE1/AE3, and SMA. Other markers including CD34 and calretinin were negative. The diagnosis was made of a retroperitoneal cystic lymphangioma (Figs. 2 and 3).

\section{Discussion}

A cystic tumor in the retroperitoneal space has many potential diagnoses, particularly when located adjacent to the pancreas, in which case the decision to operate is more difficult [4]. Lymphangiomas generally require only cystectomy; pancreatic tumors, on the other hand, usually require pancreatectomy, which is a far more involved operation.
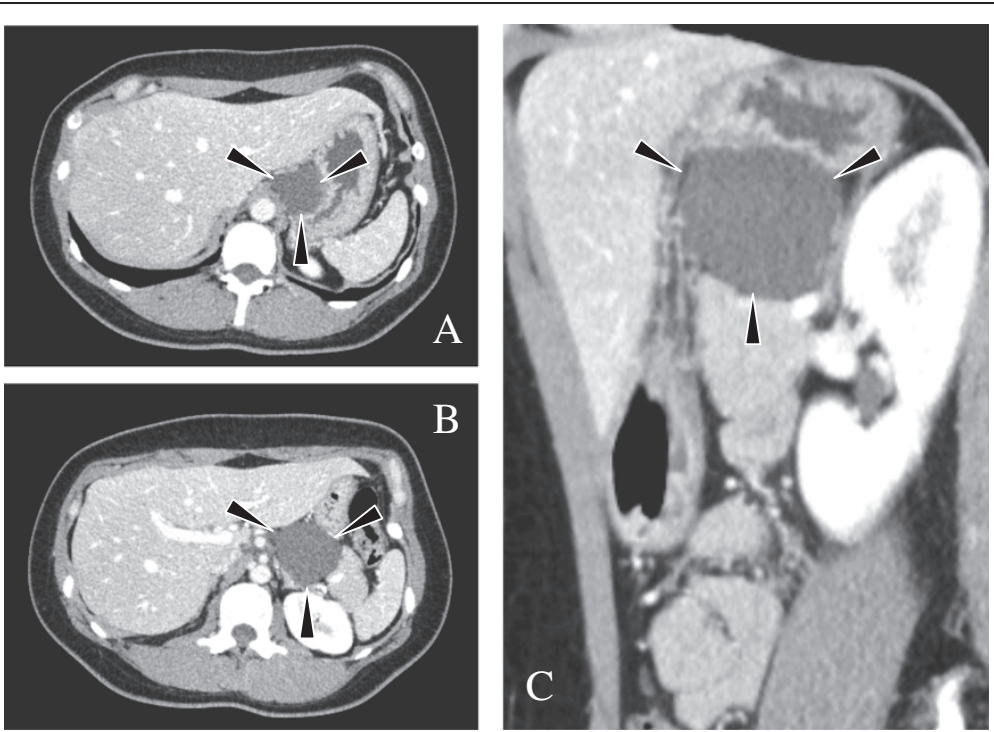

Fig. 1 Preoperative contrast-enhanced computed tomography images. $\mathbf{a}$ and $\mathbf{b}$ are axial images. $\mathbf{c}$ is a sagittal image. The arrows show the cystic tumor. The wall is not enhanced and the tumor is abutting the lesser curvature of the stomach (a and $\mathbf{c}$ ), and the body of the pancreas (b and $\mathbf{c}$ ) 

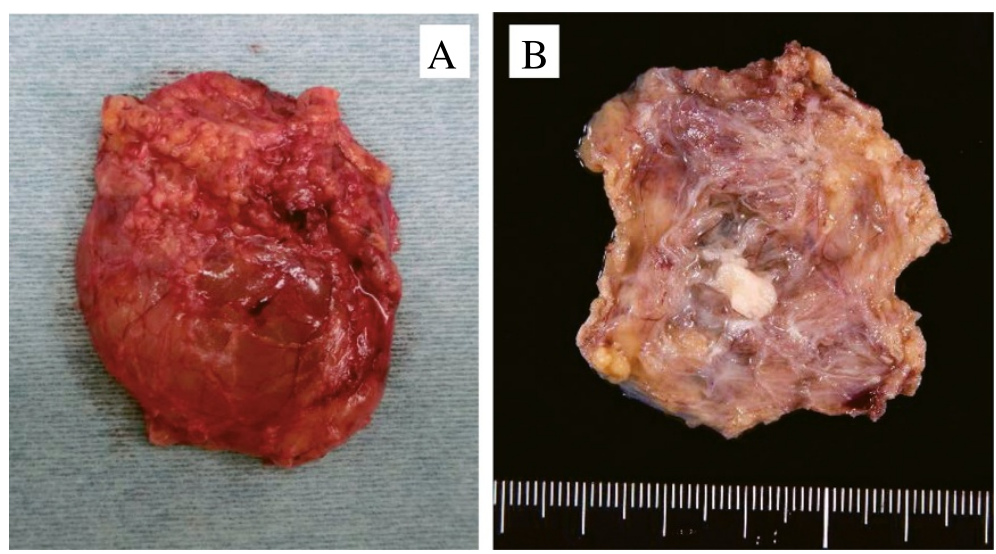

Fig. 2 a Resected specimen, gross view. Cyst fluid was serous and clear. $\mathbf{b}$ Internal aspect of the formalin-fixed specimen

Lymphangiomas are benign lesions characterized by proliferating lymphatic vessels, and are classified as either hamartomas or lymphangiectasis. Since lymphangiomas are tumors that develop before birth, in most cases they are discovered when patients are young [6]. Adult cases are rare and retroperitoneal lesions are rarer still [4]. In the present case, the tumor was located in the retroperitoneal space and abutting the pancreas; we were therefore unable to make a preoperative differential diagnosis between pancreatic tumors, such as a pancreatic pseudocyst or a mucinous cystic neoplasm, and a lymphangioma based on radiology alone.

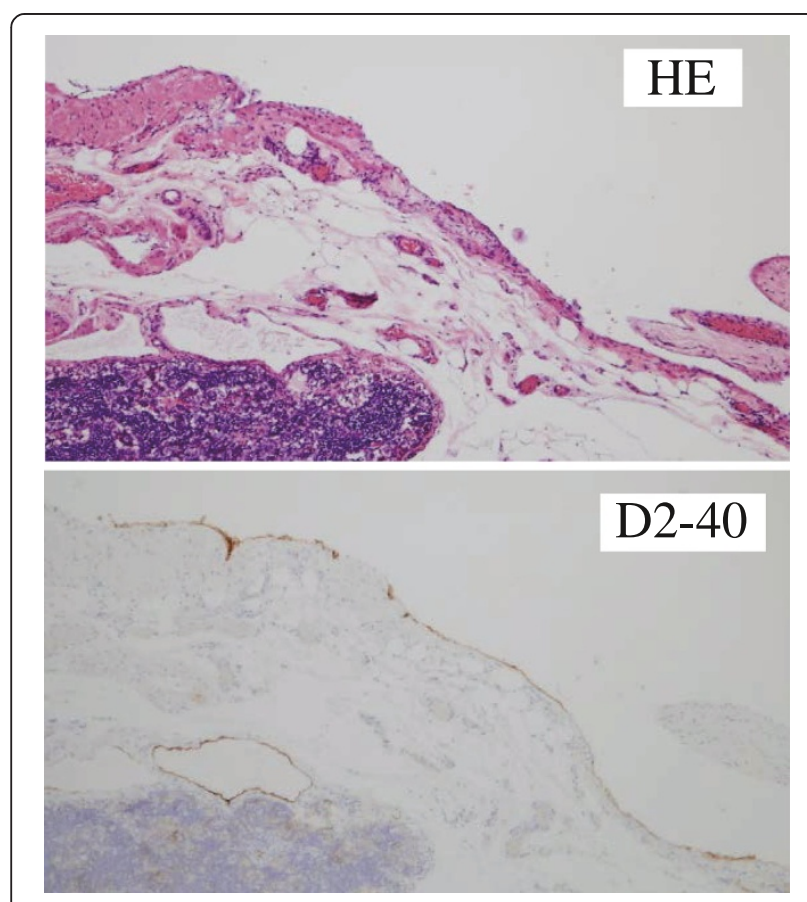

Fig. 3 Hematoxylin and eosin staining and immunostaining for D2-40. Internal aspect of the wall is lined with D2-40-positive endothelial cells
Makni et al. espouse the concept that an extended resection should not be performed for benign lymphangiomas [2]. In the last 10 years (since 2005), 31 cases of retroperitoneal lymphangioma were reported. Cystectomy was performed in 21 cases, pancreaticoduodenectomy in one case, distal pancreatectomy with splenectomy in four cases, adrenalectomy in two cases, and miscellaneous abdominal operation in one case (two reports did not describe details of the surgical procedure). If surgery is undertaken for a retroperitoneal mass of unknown cell origin, the surgeon must be able to modify the extent of surgery based on the nature of the tumor as observed intraoperatively in an attempt to preserve the integrity of any adjacent organs. To that end, a laparoscopic approach is useful for diagnosing and sometimes treating such lesions.

The first report of laparoscopic resection for retroperitoneal lymphangioma was published by Targaroma et al. in 1994 [7]. Subsequently, another case was reported in the setting of the advancement of laparoscopic techniques and instruments. To date, there are only eight reports describing ten cases with laparoscopic resection and two reports describing retroperitoneoscopic resection for retroperitoneal lymphangiomas in adult patients [2, 7-15]. Table 1 lists all of the reported cases, including our report. The median age was 45 years old (range; 25-79) and median size of the tumor was $10 \mathrm{~cm}$ (range; $3-18$ ). The male to female ratio was 2:11. These reports showed the usefulness of a laparoscopic approach for retroperitoneal lymphangiomas; this approach can also be useful for diagnosis. For example, when the tumor is located on the superior border of the pancreas in the retroperitoneal space, as in our case, we can resect the tumor using the same technique used for lymph node dissection for gastric cancer without damaging the tumor and pancreas. In patients with lymphangiomas, more precise techniques than those of lymph node dissection are required because the wall of the lymphangioma is weak and fragile. 
Table 1 Previous reports on laparoscopic/retroperitoneoscopic resection for retroperitoneal cystic lymphangiomas

\begin{tabular}{lllllll}
\hline Author & Year & Age & Gender & Symptom & Size (cm) & Approach \\
\hline Tagarona et al. [7] & 1994 & 45 & F & Abdominal pain and palpable mass & 12 & Laparoscopic \\
Tsukamoto et al. [14] & 2003 & 36 & F & Hypochondralgia and back pain & 11 & Laparoscopic \\
Celia et al. [12] & 2007 & 25 & F & Abdominal pain & 7 & 6 \\
Trindade et al. [13] & 2007 & 68 & M & Abdominal pain & 10 & Laparoscopic \\
Kasza et al. [15] & 2010 & 52 & M & Back pain & 18 & Laparoscopic \\
Yagihashi et al. [10] & 2011 & 38 & F & Hypochondralgia & Retroperitoneoscopic \\
Makni et al. [2] & 2012 & 37 & F & Abdominal pain & 11 & Laparoscopic \\
& & 48 & F & None (incidental finding) & 10 & Laparoscopic \\
Black et al. [9] & 2013 & 66 & F & Abdominal pain & 5 & Laparoscopic \\
Liu et al. [1 1] & 2013 & 45 & F & None (incidental finding) & 3 & Laparoscopic \\
Jung et al. [8] & 2014 & 79 & F & Epigastric discomfort, poor oral intake & 13 & Retroperitoneoscopic \\
Present case & 2015 & 30 & F & None (incidental finding) & Laparoscopic \\
\hline
\end{tabular}

For this purpose, the magnification inherent in laparoscopy makes for easier identification.

\section{Conclusions}

A retroperitoneal lymphangioma in close approximation to the pancreas is easily and safely resected laparoscopically. In addition to the therapeutic significance in differentiating between a cystic lymphangioma in close approximation to the body of the pancreas and a pancreatic cystic neoplasm, the laparoscopic approach is feasible and effective.

\section{Consent}

Written informed consent was obtained from the patient for publication of this case report and any accompanying images. A copy of the written consent is available for review by the Editor-in-Chief of this journal.

\section{Additional file}

Additional file 1: Laparoscopic resection of retroperitoneal lymphangioma around the pancreas. Movie file of our procedure. (M4V $20211 \mathrm{~kb}$ )

\section{Competing interests}

The authors declare that they have no competing interests.

\section{Authors' contributions}

All authors were involved in the preparation of this manuscript. TS and YM collected the data and drafted the manuscript. TS, YM, KS, MM, HM, and HT contributed to management of the patient and revised the manuscript. All authors read and approved the final manuscript.

Received: 23 June 2015 Accepted: 5 November 2015

Published online: 10 December 2015

\section{References}

1. Colovic RB, Grubor NM, Micev MT, Atkinson HD, Rankovic VI, Jagodic MM Cystic lymphangioma of the pancreas. World J Gastroenterol. 2008;14:6873-5.
2. Makni A, Chebbi F, Fetirich F, Ksantini R, Bedioui H, Jouini M, et al. Surgical management of intra-abdominal cystic lymphangioma. Report of 20 cases. World J Surg. 2012;36:1037-43.

3. Singh S, Baboo ML, Pathak IC. Cystic lymphangioma in children: report of 32 cases including lesions atrrare sites. Surgery. 1971;69:947-51.

4. Paal E, Thompson LD, Heffess CS. A clinicopathologic and immunohistochemical study of ten pancreatic lymphangiomas and a review of the literature. Cancer. 1998:82:2150-8.

5. Mansour NM, Salyers Jr WJ. Recurrence of a pancreatic cystic lymphangioma after diagnosis and complete drainage by endoscopic ultrasound with fine-needle aspiration. JOP. 2013;14:280-2.

6. Bhavsar T, Saeed-Vafa D, Harbison S, Inniss S. Retroperitoneal cystic lymphangioma in an adult: a case report and review of the literature. World J Gastrointest Pathophysiol. 2010;1:171-6.

7. Targarona EM, Moral A, Sabater L, Martinez J, Luque P, Trias M. Laparoscopic resection of a retroperitoneal cystic lymphangioma. Surg Endosc. 1994;8: 1425-6.

8. Jung HI, Ahn T, Son MW, Kim Z, Bae SH, Lee MS, et al. Adrenal lymphangioma masquerading as a pancreatic tail cyst. World J Gastroenterol. 2014;20:13195-9.

9. Black T, Guy CD, Burbridge RA. Retroperitoneal cystic lymphangioma diagnosed by endoscopic ultrasound-guided fine needle aspiration. Clin Endosc. 2013;46:595-7.

10. Yagihashi $Y$, Kato $K$, Nagahama $K$, Yamamoto $M$, Kanamaru $H$. A case of laparoscopic excision of a huge retroperitoneal cystic lymphangioma. Case Rep Urol. 2011:2011:712520.

11. Liu B, Li Y, Wang S. Adrenal lymphangioma removed by a retroperitoneoscopic procedure. Oncol Lett. 2013;5:539-40.

12. Celia A, Breda G. Laparoscopic excision of a retroperitoneal cystic lymphangioma: an insidious case. J Laparoendosc Adv Surg Tech A. 2007;17:315-6.

13. Trindade EN, Trindade MR, Boza JC, Von Diemen $V$, Ilgenfritz RB. Laparoscopic excision of a retroperitoneal cystic lymphangioma in an elderly patient. Minerva Chir. 2007:62:145-7.

14. Tsukamoto T, Tanaka S, Yamamoto T, Kakinoki E, Uemichi A, Kubo S, et al. Laparoscopic excision of a retroperitoneal cystic lymphangioma: report of a case. Surg Today. 2003;33:142-4.

15. Kasza J, Brody FJ, Khambaty F, Vaziri K, Wallace B. Laparoscopic resection of a retroperitoneal cystic lymphangioma in an adult. Surg Laparosc Endosc Percutan Tech. 2010;20:e114-116. 